\title{
Models of frequency preferences of prefrontal cortical neurons
}

\author{
Arthur R. Houweling ${ }^{a, b, *}$, Rashmi H. Modia , Paul Ganter ${ }^{a}$, \\ Jean-Marc Fellous ${ }^{a}$, Terrence J. Sejnowski ${ }^{\mathrm{a}, \mathrm{c}}$ \\ ${ }^{2}$ Computational Neurobiology Laboratory, The Salk Institute, 10010 N Torrey Pines Roads, La Jolla, \\ CA 92037, USA \\ 'Program in Neurosciences, University of California, San Diego, La Jolla, CA 92093, USA \\ 'Department of Biology, University' of California, San Diego, La Jolla, CA 92093, USA
}

\begin{abstract}
The reliability of spike trains generated by sinusoidal current injections in prefrontal cortical pyramidal cells and interneurons depends strongly on the input frequency. We constructed computational models in order to study how cellular properties affect reliability. The models reproduced the main experimental findings: subthreshold oscillations, resonance and reliability of spike timing. The amplitude of intrinsic noise in the model determined the number of reliable frequency bands. In addition, the frequency content of the noise did not affect reliability. (C) 2001 Elsevier Science B.V. All rights reserved.
\end{abstract}

\section{Introduction}

Prefrontal cortical pyramidal cells show sub-threshold oscillations and intrinsic preferences for restricted input frequency ranges $[1,3,4]$. In addition, spike trains generated by sinusoidal current injections are most reliable in some input frequency bands [1]. The cellular properties responsible for the restricted reliability are unclear. Here we construct computational models matched to our experimental data in order to study how different cellular properties affect reliability. In particular, we focus on the influence of intrinsic noise.

\footnotetext{
* Corresponding author. Computational Neurobiology Laboratory, The Salk Institute, $10010 \mathrm{~N}$ Torrey Pines Roads, La Jolla, CA 92037, USA. Tel.: + 1-619-453-4100-1463.

E-mail address: arthur@salk.edu (A. R. Houweling).
} 


\section{Methods}

Experimental procedures are described in detail elsewhere [1]. Briefly, coronal slices of rat prelimbic and infralimbic areas of prefrontal cortex were obtained from 2-4 weeks old rats. Whole cell recordings were made from regular spiking layer five pyramidal cells $(n=13)$ at $30-32^{\circ} \mathrm{C}$. In all experiments, synaptic transmission was blocked by APV, CNQX and biccuculine. Sub- and suprathreshold responses of cells were characterized for sinusoidal current injections at different frequencies and amplitudes. The minima of all injected sinusoidal currents occurred at zero. Spike count and reliability values were determined for a $1 \mathrm{~s}$ period following a $1 \mathrm{~s}$ transient period after stimulus onset. Reliability values for individual cells were grouped on the basis of their responses to 'low' and 'high' amplitude current injections. The 'low' amplitude was defined as the amount of current needed to evoke a single spike at $0.5 \mathrm{~Hz}$ about once every three trials, the 'high' amplitude was twice this amplitude. All results are given as mean \pm standard deviation.

The reliability measure is similar to measures used in previous studies $[5,6]$. First, the spike histogram calculated from 10-15 trials of a particular sinusoidal current injection was smoothed using a window of $6 \mathrm{~ms}$. Bins of the smoothed histogram exceeding a threshold value of $50 \%$ of the number of trials defined a reliable event. Reliability is the ratio of the number of spikes in reliable events to the total number of spikes in the spike histogram, which gives a value between 0 and 1 . Reliability values for the model are given as the mean \pm standard deviation of 10 series of 15 trials.

The single compartment pyramidal (PY) cell model was based on a model introduced by Golomb and Amitai [2]. The model included a leak current, sodium and potassium currents for action potential generation, a persistent sodium current $I_{\mathrm{NaP}}$, an A-type potassium current $I_{\mathrm{A}}$ and a slow potassium current $I_{\mathrm{Ks}}$. An H-type mixed cation current $I_{\mathrm{H}}[7]$ was added to reproduce the sag in membrane potential in response to hyperpolarizing current injections observed experimentally. The PY cell responded with adapting spike trains to depolarizing step current injections. A noise current consisting of white noise convolved with an alpha function with time constant $\tau=3 \mathrm{~ms}$ was added to the model to calculate reliability values. The RMS value of the convolved noise $(\sigma=0.1 \mathrm{nA})$ was set to approximately match peak reliability values of the sample cell in Fig. 1B/C. The actual level of injected noise was scaled by a sigmoidal function of membrane potential $1 /(1+\exp [(65-V) / 2.5])$ to reduce membrane potential fluctuations at the resting potential $(-71 \mathrm{mV})$.

The multiple compartment pyramidal cell model was based on a reconstructed pyramidal cell [Turner D.A., Southampton Archive cell n 400]. The model was endowed with the same ionic currents as for the single compartment PY cell. Currents were placed at the soma, while dendrites were passive with a membrane capacitance double to that of the soma to account for dendritic spines. The interneuron (IN) model included fast sodium and potassium currents responsible for action potential generation [8] and an M-type potassium current [9]. The passive properties of the model were tuned to reproduce experimental recordings obtained in prefrontal cortex. All simulations were run using NEURON [10]. 


\section{Results}

\subsection{Experiments}

The peak membrane potential of prefrontal cortical pyramidal cells depended on the frequency of subthreshold sinusoidal current injections (Fig. 1A). The amplitude of the membrane potential was largest at input frequencies that were similar to the dominant frequencies of spontaneous subthreshold oscillations observed in these cells when depolarized by a constant pulse ( $\approx 4 \mathrm{~Hz}$, not shown). When the amplitude of the injected current was increased just above spike threshold ('low' current input), spikes first appeared at theta range frequencies (Fig. 1B, black dots). Doubling the amplitude of injected current ('high' current input) tripled the maximal firing rate and shifted the peak spike rate to input frequencies around $15 \mathrm{~Hz}$ (Fig. 1B, open circles). Next, we calculated reliability values for the spike trains generated by the different sinusoidal inputs. The 'low' current inputs resulted in zero reliability at all input frequencies. Using a reliability measure with a larger integration window of $20 \mathrm{~ms}$ (similar to [5]) a peak reliability of 0.41 at $4 \mathrm{~Hz}$ was revealed. The 'high' current inputs yielded non-zero reliability values in the range of $5-20 \mathrm{~Hz}$, with a peak reliability of 0.47 at $11 \mathrm{~Hz}$ (Fig. 1C, open circles). Note that inputs at this frequency generated on average one spike per cycle (Fig. 1B). Group data displayed reliability peaks at similar input frequencies (Fig. 1C), around $6 \mathrm{~Hz}$ for 'low' amplitude (black squares) and around $15 \mathrm{~Hz}$ for 'high' amplitude (open squares) current injections. Note that peak reliability increased with larger current injections.

Interneurons were capable of generating reliable spike trains at input frequencies up to $40-60 \mathrm{~Hz}$ (not shown). In this frequency range cells fired close to one spike per input cycle.
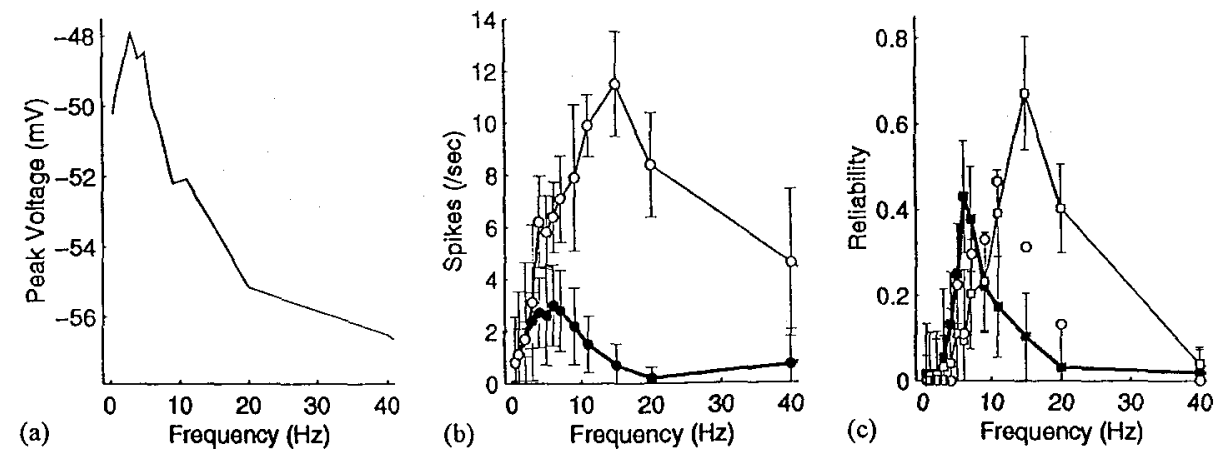

Fig. 1. Experimental responses of pyramidal cells to sinusoidal inputs. (A) Maximum membrane potential in response to subthreshold current injections of constant amplitude at different frequencies in a single cell. (B) Number of spikes per second for 'low' (black dots) and 'high' current (open circles) inputs from another cell. (C) Reliability values for 'high' current inputs from the same cell as in B (open circles) and reliability group data for 'low' current (black squares) and 'high' current (open squares) injections. 


\subsection{Models}

Both multiple- and single-compartment pyramidal cell models displayed a resonance for subthreshold current injections similar to experimental data. The peak of the voltage response to current injection is shown as a function of input frequency for the single compartment PY model (Fig. 2A). The subthreshold resonance appeared at frequencies around 3-4 Hz. Moreover, the PY cell displayed subthreshold oscillations in this frequency range in response to step current injections (not shown). The multiple compartment model showed a reduction of the mean somatic depolarization with more distal input locations, which led to a shift of the resonance frequency to lower values (not shown). In the following we limited our analysis to the single compartment PY cell model.

For current injections above but close to the firing threshold $(0.1 \mathrm{nA})$ the PY cell fired action potentials only at input frequencies between 2 and $9 \mathrm{~Hz}$ (Fig. $2 \mathrm{~B}$, asterisks). This was similar to the suprathreshold resonance of pyramidal cells (Fig. 1B). The incorporation of noise in the PY cell model caused action potentials to occur outside this frequency range (Fig. $2 \mathrm{~B}$, open squares). Stronger current injections evoked a maximum number of spikes at input frequencies with one spike per input cycle (Fig. 2B, circles and dots). Next, we calculated reliability values for the spike trains generated by the different sinusoidal inputs. The reliability values for the $0.1 \mathrm{nA}$ current injections were zero for all input frequencies, like those for the 'low' current injection in the pyramidal cell of Fig. 1B. Using the modified reliability measure ( $20 \mathrm{~ms}$ window) we obtained a peak reliability of 0.37 at $4 \mathrm{~Hz}$ for the model, similar to the peak reliability of 0.41 at $4 \mathrm{~Hz}$ for the experiment. Stronger current injections generated reliable spike trains for frequencies in small bands around $7 \mathrm{~Hz}(0.2 \mathrm{nA})$ or $10 \mathrm{~Hz}(0.3 \mathrm{nA}$ ) (Fig. 2C). Note that the reliability (and spike rate) curves for the $0.3 \mathrm{nA}$ current injections were comparable to those for the experimental 'high' current injections (Fig. 1B/C, circles). Peak reliability increased gradually from 0.10 at $0.15 \mathrm{nA}$
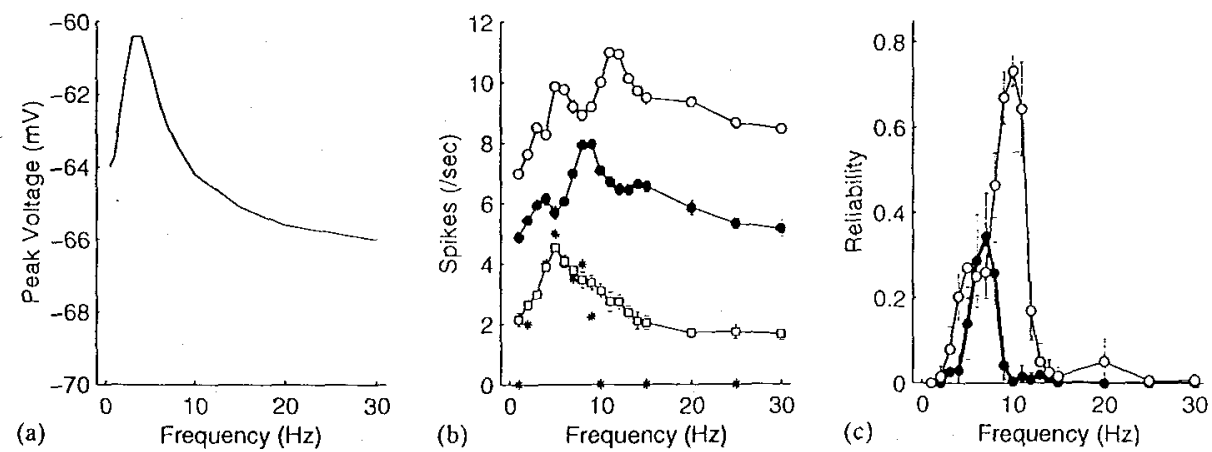

Fig. 2. Model PY cell responses to sinusoidal inputs. (A) Peak voltage deflection for subthreshold inputs of constant amplitude at different frequencies. (B) Number of spikes per second for inputs with mean current of $0.1 \mathrm{nA}$ (open squares), $0.2 \mathrm{nA}$ (black dots) and $0.3 \mathrm{nA}$ (open circles), and number of spikes for $0.1 \mathrm{nA}$ inputs in the deterministic model (asterisks). (C) Reliability values for inputs with mean current of $0.2 \mathrm{nA}$ (black dots) and $0.3 \mathrm{nA}$ (open circles). 

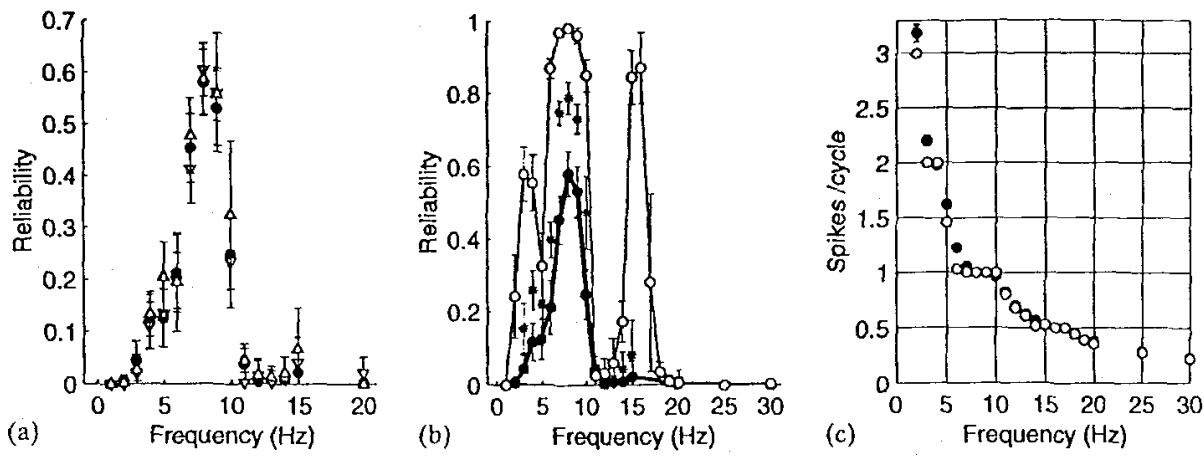

Fig. 3. Effects of power and frequency content of noise on PY cell reliability for sinusoidal inputs. (A) Reliability values when the noise was generated with an alpha filter with a time constant of $0.1 \mathrm{~ms}$ (downward triangles), $3 \mathrm{~ms}$ (circles) and $25 \mathrm{~ms}$ (upward triangles). (B) Reliability values for a level of noise of $\sigma=0.1 \mathrm{nA}$ (black dots), $0.075 \mathrm{nA}$ (asterices) and $0.04 \mathrm{nA}$ (open circles). (C) Spikes per cycle for different input frequencies with a level of noise of $\sigma=0.1 \mathrm{nA}$ (black dots) and $0.04 \mathrm{nA}$ (open circles). The mean current was $0.25 \mathrm{nA}$ for all current injections.

to 0.96 at $0.5 \mathrm{nA}$ (not shown). The corresponding input frequencies shifted from 6 to $16 \mathrm{~Hz}$, respectively, and generated on average one spike per cycle.

Next, we considered how the properties of the noise affected reliability in the PY cell model (Fig. 3). In particular, we changed the frequency content and the power of the noise and examined reliability values for $0.25 \mathrm{nA}$ current injections. The frequency content of the noise was changed by taking different time constants $(\tau)$ for the alpha filter while keeping the total power constant. The resulting reliability values were similar at all frequencies for different values of $\tau$ (Fig. 3A). As expected, the power of the noise had a large impact on reliability (Fig. 3B). At $75 \%$ of the original noise level, reliability values were most strongly increased in the peak reliability frequency range around $8 \mathrm{~Hz}$ (Fig. 3B, asterisks). When the noise level was reduced to $40 \%$, interestingly, two additional frequency bands developed strong reliability (Fig. 3B, open circles). These bands included frequencies at $3-4$ and $15-16 \mathrm{~Hz}$ corresponding to regimes with two spikes per cycle and one spike per two cycles, respectively (Fig. 3C).

Simulations were also performed to reproduce the experimental results obtained for interneurons. We found that our simple model was able to reproduce some of the firing characteristics observed experimentally, including the high reliability of spike trains generated by inputs in the gamma frequency range (not shown). Further work is currently aimed at refining the interneuron model.

\section{Discussion}

The pyramidal cell model reproduced most of the resonance, subthreshold oscillation and spike rate characteristics of experimental cells. When noise was added, the model cell produced reliability curves similar to those measured experimentally. In 
particular, we observed that the most reliable spike trains were generated in a small frequency band for which inputs usually generated one spike per input cycle. When this frequency band occurred at low frequencies, peak reliability was low because of the low input amplitude to noise ratio. With reduced levels of noise two additional narrow frequency bands appeared for which reliable spike trains were generated. These spike trains had either two spikes per input cycle, or one spike per two input cycles. These results suggest that reliable spike trains occur if the input frequency is a small integer multiple of the spike frequency and vice versa. However, this may be a necessary but not a sufficient condition. For example, in the model with all but the leak and action potential conductances set to zero, peak reliability occurred when one spike per cycle was elicited. In contrast, one spike per cycle also occurred at other frequencies (but same current amplitude) with close to zero reliability values (not shown). The observation that the frequency content of the noise does not influence reliability values indicates that the particular noise implementation may not be crucial for the study of reliability in our model. The results from this study suggest a method for estimating the level of intrinsic noise in neurons through the measurement of reliability values.

\section{Acknowledgements}

This work was supported by the Howard Hughes Medical Institute.

\section{References}

[1] J-M. Fellous, A.R. Houweling, R.H. Modi, R.P.N. Rao, P.H.E. Tiesinga, T.J. Sejnowski, Frequency dependence of spike timing reliability in cortical pyramidal cells and interneurons, J. Neurophysiol. 85 (4) (2001) 1782-1787.

[2] D. Golomb, Y. Amitai, Propagating neuronal discharges in neocortical slices: computational and experimental study, J. Neurophysiol. 78 (3) (1997) 1199-1211.

[3] Y. Gutfreund, Y. Yarom, I. Segev, Subthreshold oscillations and resonant frequency in guinea-pig cortical neurons: physiology and modelling, J. Physiol. 483 (3) (1995) 621-640.

[4] R.R. Llinas, A.A. Grace, Y. Yarom, In vitro neurons in mammalian cortical layer 4 exhibit intrinsic oscillatory activity in the 10- to 50-Hz frequency range, Proc. Natl. Acad. Sci. USA 88 (3) (1991) 897-901.

[5] Z.F. Mainen, T.J. Sejnowski, Reliability of spike timing in neocortical neurons, Science 268 (5216) (1995) 1503-1506.

[6] L.G. Nowak, M.V. Sanchez-Vives, D.A. McCormick, Influence of low and high frequency inputs on spike timing in visual cortical neurons, Cerebral Cortex 7 (6) (1997) 487-501.

[7] W.J. Spain, P.C. Schwindt, W.E. Crill, Anomalous rectification in neurons from cat sensorimotor cortex in vitro, J. Neurophysiol. 57 (5) (1987) 1555-1576.

[8] R.D. Traub, R. Miles, G. Buzsaki, Computer simulation of carbachol-driven rhythmic population oscillations in the CA3 region of the in vitro rat hippocampus, J. Physiol. 451 (1992) 653-672.

[9] W. Yamada, C. Koch, P.R. Adams, Multiple channels and calcium dynamics, in: C. Koch, I. Segev (Eds.), Methods in Neuronal Modeling: From Synapses to Networks, MIT Press, Cambridge, MA, 1989, pp. 97-133.

[10] M.L. Hines, N.T. Carnevale, The NEURON simulation environment, Neural Computation 9 (1997) 1179-1209. 


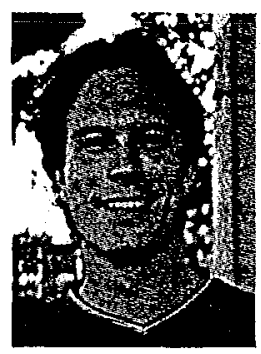

Arthur Houweling is a doctoral candidate in the Computational Neurobiology Laboratory at the Salk Institute and the Program in Neurosciences at the University of California, San Diego. He received his M.S. in biology from the University of Leiden, the Netherlands in 1994. His thesis research focuses on the mechanisms underlying sleep oscillations in thalamocortical systems.

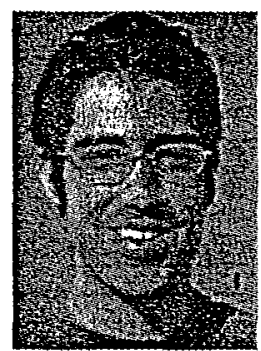

Rashmi Modi is a master's candidate in the Computational Neurobiology Laboratory at the Salk Institute in the Biology Graduate Program at the University of California, San Diego. He received his B.S. in neuroscience from U.C.S.D. in 1999. His thesis is on oscillation-based behavior in cortical interneurons.

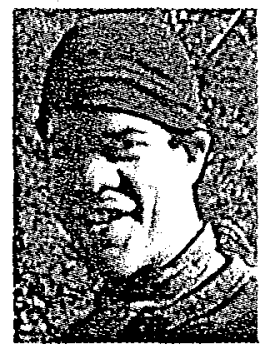

Paul Ganter did his undergraduate studies in physics at the L.M.U. Munich. Germany. He then moved to Cambridge, UK, where he specialized in Dynamical Systems theory and Fluid Dynamics. Being offered a place as a Wellcome Trust Prize student in a 4-year interdisciplinary program in Neuroscience he moved to Oxford, UK. There he is currently working with Ole Paulsen in the Department of Physiology. His main interests are modulation of synaptic transmission by metabotropic glutamate receptors and resonance phenomena in different types of cells in the hippocampal network using a combined approach integrating in vitro experiments and computational modeling. This work is conducted in collaboration with Terrence Sejnowski's laboratory (Salk Institute, CA).

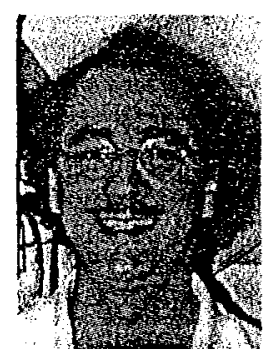

Jean-Marc Fellous did his undergraduate studies in Marseille, France. Graduate studies included a Master's degree in artificial intelligence (Paris VI University, France), and a Ph.D. in computer science with an emphasis on brain theory and neural networks at the University of Southern California, Los Angeles. Postdoctoral work included slice neurophysiology and neural modeling in the laboratories of John Lisman (Brandeis University) and Terrence Sejnowski (Salk Institute). J-M.F. is currently a Howard Hughes Institute Research Associate in the Computational Neurobiology Laboratory. His interests include the computational roles of neuromodulation and rhythmogenesis. 


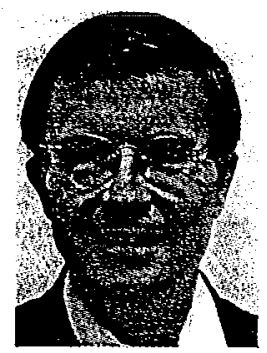

Terrence Sejnowski is an Investigator with the Howard Hughes Medical Institute and a Professor at The Salk Institute for Biological Studies where he directs the Computational Neurobiology Laboratory. He is also Professor of Biology at the University of California, San Diego, where he is Director of the Institute for Neural Computation. Dr. Sejnowski received a B.S. in physics from the CaseWestern Reserve University, an M.A. in physics from Princeton University, and a Ph.D. in physics from Princeton University in 1978. In 1988, Dr. Sejnowski founded Neural Computation, published by the MIT Press. He is also the President of the Neural Information Processing Systems Foundation. The longrange goal of Dr. Sejnowski's research is to build linking principles from brain to behavior using computational models. This goal is being pursued with a combination of theoretical and experimental approaches at several levels of investigation ranging from the biophysical level to the systems level. 